\title{
The Monoaminergic Modulation of Sensory-Mediated Aversive Responses in Caenorhabditis elegans Requires Glutamatergic/Peptidergic Cotransmission
}

\author{
Gareth Harris, Holly Mills, Rachel Wragg, Vera Hapiak, Michelle Castelletto, Amanda Korchnak, \\ and Richard W. Komuniecki \\ Department of Biological Sciences, University of Toledo, Toledo, Ohio 43606-3390
}

\begin{abstract}
Monoamines and neuropeptides interact to modulate behavioral plasticity in both vertebrates and invertebrates. In Caenorhabditis elegans behavioral state or "mood" is dependent on food availability and is translated by both monoaminergic and peptidergic signaling in the fine-tuning of most behaviors. In the present study, we have examined the interaction of monoamines and peptides on C. elegans aversive behavior mediated by a pair of polymodal, nociceptive, ASH sensory neurons. Food or serotonin sensitize the ASHs and stimulate aversive responses through a pathway requiring the release of $n l p$-3-encoded neuropeptides from the ASHs. Peptides encoded by $n l p-3$ appear to stimulate ASH-mediated aversive behavior through the neuropeptide receptor-17 (NPR-17) receptor. $n l p-3$ - and $n p r-17$-null animals exhibit identical phenotypes and animals overexpressing either $n l p-3$ or $n p r-17$ exhibit elevated aversive responses off food that are absent when $n l p-3$ or $n p r-17$ are overexpressed in $n p r-17$ - or $n l p$-3-null animals, respectively.

ASH-mediated aversive responses are increased by activating either $\mathrm{G} \alpha_{\mathrm{q}}$ or $\mathrm{G} \alpha_{\mathrm{s}}$ in the ASHs, with $\mathrm{G} \alpha_{\mathrm{s}}$ signaling specifically stimulating the release of $n l p$-3-encoded peptides. In contrast, octopamine appears to inhibit 5-HT stimulation by activating $\mathrm{G} \alpha_{\mathrm{o}}$ signaling in the ASHs that, in turn, inhibits both $\mathrm{G} \alpha_{\mathrm{s}}$ and $\mathrm{G} \alpha$, signaling and the release of $n l p$-3-encoded peptides. These results demonstrate that serotonin and octopamine reversibly modulate the activity of the ASHs, and highlight the utility of the C. elegans model for defining interactions between monoamines and peptides in individual neurons of complex sensory-mediated circuits.
\end{abstract}

\section{Introduction}

Mood disorders, such as depression, are among the most common mental illnesses and are often treated with drugs that elevate monoamine levels, leading to the "monoamine hypothesis" of depression. In addition, other signaling cascades, including those mediated by peptides, are also important (Lanni et al., 2009). For example, substance $\mathrm{P}$, neurokinin A, corticotropin-releasing factor, neuropeptide $\mathrm{Y}$, and galanin have been identified as possible candidates for antidepressant development. However, given the complexity of the vertebrate nervous system, a mechanistic understanding of monoaminergic/peptidergic interactions has been limited, encouraging the development of invertebrate model systems (Nässel et al., 2008). Caenorhabditis elegans has only 302 neurons and the well defined neuronal pathways modulating its sensory-mediated locomotory behaviors are excellent models to examine monoaminergic/peptidergic interactions in the modulation of individual neurons or neuronal circuits. Importantly,

Received Jan. 28, 2010; revised April 16, 2010; accepted April 20, 2010.

This work was supported by National Institutes of Health Grant Al-145147 awarded to R.W.K. and funds from the Joan L. and Julius H. Jacobson Biomedical Professorship. We thank Drs. Bruce Bamber and Robert Steven for critical discussion of the manuscript, Dr. S. Mitani at the National Bioresources Project and the Caenorhabditis elegans Genetics Center, which is supported by the National Institutes of Health National Center for Research Resources, for strains.

Correspondence should be addressed to Richard W. Komuniecki, Department of Biological Sciences, University of Toledo, 2801 W. Bancroft Street, Toledo, OH 43606-3390. E-mail: rkomuni@uoft02.utoledo.edu.

DOI:10.1523/JNEUROSCI.0497-10.2010

Copyright $\odot 2010$ the authors $\quad 0270-6474 / 10 / 307889-11 \$ 15.00 / 0$ most C. elegans monoamine receptors are at least partially characterized and most have clear orthologs in mammals (Chase and Koelle, 2007; Komuniecki et al., 2004).

In C. elegans, the polymodal, nociceptive ASH sensory neurons are essential for avoidance responses to a variety of sensory cues, with the ASH-mediated circuit proposed as a model for pain sensation in mammals (Kaplan and Horvitz, 1993; Bargmann and Kaplan, 1998; Sambongi et al., 1999; Hilliard et al., 2004; Walker et al., 2009). However, although we have a rudimentary knowledge of ASH activation; for example, a transient receptor potential vanilloid (TRPV) channel contributes to ASHmediated aversive responses; little is known about how behavioral state modifies this sensory machinery (Tobin et al., 2002). In C. elegans, behavioral state or "mood" is defined largely by the availability of food and is translated by both monoaminergic and peptidergic signaling in the modulation of key behaviors. For example, serotonin (5-HT) stimulates feeding, egg-laying and complex behaviors, including chemosensation and aversive learning. In contrast, tyramine (TA) and octopamine (OA), the invertebrate equivalents of epinephrine and norepinephrine, independently oppose the actions of 5-HT (Alkema et al., 2005; Wragg et al., 2007; Pirri et al., 2009). Monoamines also modulate sensory-mediated locomotory behaviors. For example, ASHmediated aversive responses are stimulated by food/5-HT and inhibited by TA/OA (Wragg et al., 2007; Harris et al., 2009). Three different 5-HT receptors, operating at different levels within the ASH-mediated circuit, are each essential for seroto- 
nergic stimulation (Harris et al., 2009). Similarly, the C. elegans genome encodes $>200$ neuropeptides and 50 peptide receptors, many with putative orthologs in mammals, but our knowledge of how individual peptides contribute to "mood," modulate individual neurons or alter behavior also is limited ( $\mathrm{Li}$ and Kim, 2008).

In the present study, we identified the G-protein signaling pathways involved in the monoaminergic modulation of the ASHs and have demonstrated that neuropeptides encoded by $n l p-3$ are essential for the serotonergic stimulation of the ASHmediated locomotory circuit. 5-HT and OA antagonistically modulate the activity state of the ASHs. These results highlight the utility of the C. elegans model for study of monoaminergic/ peptidergic interactions in individual neurons of complex, sensory-mediated circuits.

\section{Materials and Methods}

Materials. All reagents were purchased from Sigma Aldrich. DMEM was purchased from Media-Tech, neurochemicals from Sigma-Aldrich, restriction enzymes from New England Biolabs and Promega, and oligonucleotide primers from Integrated DNA Technologies. A C. elegans cDNA pool was purchased from OriGene Technologies, and additional cDNA pools were constructed from mixed stage mRNA using standard techniques. Green fluorescent protein (GFP) expression vectors were obtained from Andy Fire (Stanford School of Medicine).

Culture and maintenance of strains. The N2 Bristol WT isolate of $C$. elegans was used for all studies. All animals were raised at $20^{\circ} \mathrm{C}$ under uncrowded conditions (Brenner, 1974). The following mutant alleles were used: acy-1(ce2)III, dgk-1(sy428)X, eat-4(ky5)III, eat-16(ce71)I, egl3(n150)V, egl-8(n488)V, egl-21(n476)IV, egl-30(js126)I, eri-1(kp3948)IV, gsa-1(ce81)I, octr-1(f14d12.6)(ok371)X, nlp-3(tm2302)X, pde-4(ce268)II, ser-5(tm2647)I, ser-5(tm2654)I, npr-17 (c06g4.5)(tm3210)III, c30f12.6 (0k1381), c43c3.2(ok1388)X, npr-9(tm1652)X, gnrr-1(ok238)I, t27d1.3 (ok1626)III, y39a3b.5(tm3082)III, t02e9.3(ok568)V, fshr-1(ok778) V, c02h7.2(ok2068)X, c48c5.1(ok1387)X, w05b5.2(tm2974)I, f14f4.1(tm2243)X, t07d10.2(tm2765)I, npr-10(ok1541)X, k10b4.4(ok3502)II, t02e9.1(ok2008)V, f02e8.2(ok2295)X, t11f9.1(ok2284) V, tkr-1(tm1765)III. All strains were obtained from the Caenorhabditis Genetics Center (University of Minnesota, Minneapolis, MN) except, f14f4.1(tm2243)X, nlp-3(tm2302)X, npr-9(tm1652)X, npr-17(tm3210)III, ser-5(tm2647)I, ser-5(tm2654)I, tkr-1(tm1765)III, t07d10.2(tm2765)I, w05b5.2(tm2974)I and y39a3b.5 (tm3082)III that were purchased from the National Bio-Resources Project (Tokyo Women's Medical University, Tokyo, Japan). Full-length egl-3p::egl-3(+) rescued animals were kindly provided by Dr. Maureen Barr (Rutgers University, Piscataway, NJ).

Behavioral assays. Assay plates (5 cm NGM plates) were prepared daily and 5-HT or OA (4 mM) were added to NGM liquid media just before pouring. Dilute 1-octanol was prepared daily using $100 \%$ ethanol (v/v) (Sulston and Hodgkin, 1988; Bargmann et al., 1993; Chao et al., 2004). Synchronized fourth-stage larvae (L4) were picked $24 \mathrm{~h}$ preassay and assays were performed at $23-25^{\circ} \mathrm{C}$. Octanol avoidance and spontaneous reversal were measured as described previously (Chao et al., 2004; Gray et al., 2005; Wragg et al., 2007; Harris et al., 2009).

Octanol avoidance. Octanol avoidance was assayed as originally described by Chao et al. (2004). Briefly, the blunt end of a hair (LoewCornell 9000 Kolinsky 8 paintbrush), taped to a toothpick, was dipped in $30 \%$ octanol and the hair placed in front of an animal exhibiting forward sinusoidal locomotion. Time to reverse was recorded and assays were terminated after $20 \mathrm{~s}$, as wild-type animals spontaneously reverse on average every $20 \mathrm{~s}$ (Zhao et al., 2003; Chao et al., 2005). For assays in the absence of food or exogenous 5-HT, well fed young adults (three to five per plate) were first transferred to intermediate nonseeded plates and left for $1 \mathrm{~min}$ to prevent any bacteria/media carry over, then transferred to NGM plates and assayed after $10 \mathrm{~min}$. For assays in the presence of food (Escherichia coli OP50) or 5-HT, animals were transferred to plates containing a thin layer of OP50 or 4 mM 5-HT and assayed after 20 and 30 min, respectively. 20 animals were examined per strain assayed per con- dition. Statistical analysis was performed using mean \pm SE and Student's $t$ test.

Spontaneous reversal. The frequency of spontaneous reversal was assayed as described previously (Tsalik and Hobert, 2003; Dernovici et al., 2006). Well-fed animals were transferred to NGM plates for $30 \mathrm{~s}$, then transferred to assay plates (food) for $1 \mathrm{~min}$ and assayed. Reversal frequency was scored as the number of times an animal reversed within 3 min (Pierce-Shimomura et al., 1999). Data were presented as a mean \pm SE $(n=3)$ and analyzed by two-tailed Student's $t$ test. $p$ values were indicated as follows: “»” $p<0.001$.

Creation of mutants. Animals containing combinations of $g f$, $1 f$ or null alleles were constructed using standard genetic techniques and confirmed by PCR. ser-5(tm2654)I animals were crossed with either egl30(js126)I, gsa-1(ce81)I or pde-4(ce268)II animals to create egl-30(js126) ser-5(tm2654)I (RWK94), gsa-1(ce81) ser-5(tm2654)I (RWK95) and ser5(tm2654)I;pde-4(ce268)II (RWK96) double mutants. nlp-3(tm2302)X animals were crossed with $p d e-4(c e 268) I I$, egl-30(js126)I or gsa-1(ce268)I animals to give pde-4(ce268)II; nlp-3(tm2302)X (RWK97), gsa-1(ce81)I; nlp-3(tm2302)X (RWK104) and egl-30(js126)I; nlp-3(tm2302)X (RWK98) double mutants, respectively. eat-4(ky5) $\mathrm{V}$ animals were crossed with ser-5(tm2654)I to create ser-5(tm2654)I; eat-4(ky5)V (RWK101). eat$4(k y 5) V$ animals were also crossed with egl-3(n150)V and $n l p-$ $3($ tm2302)X to generate eat-4(ky5)V;egl-3(n150)V (RWK102) and eat-4(ky5)V;nlp-3(tm3202)X (RWK103) double mutants, respectively. All mutant animals were screened by PCR with primers flanking the deletions to confirm the crosses. To confirm that the mutant animals were homozygous for the deletion, a second PCR was conducted using the forward primer flanking the deletion and a reverse primer internal for the deletion. Homozygous mutants produced no PCR product in this reaction. All mutant animals were backcrossed with the N2 Bristol strain at least four times before use in assays or crosses. The following strains were backcrossed with $\mathrm{N} 2$ Bristol strain before use in the generation of the double mutants, eat-4(ky5)4X (RWK22), egl-3(n150)4X (RWK23), $n l p-3(t m 2302) 4 X$ (RWK24) and ser-5(tm2654)5X (RWK51).

Creation of cell-specific RNAi constructs. Neuron selective/specific RNAi transgenes were constructed as described by Esposito et al. (2007). (Sense and antisense primers and a list of strains are available in supplemental Table S2, available at www.jneurosci.org as supplemental material.) For the creation of RNAi transgenes, a neuron-selective promoter ( $s r a-6 p$ for ASH/ASI; $g p a-4 p$ for ASI) was fused to exon rich regions of the target gene. Exon rich regions were amplified using a forward and reverse primer to create template A from either cDNA or genomic DNA by PCR-fusion, as previously described (Hobert, 2002). Neuron-selective promoters were amplified using a forward primer with reverse (sense) or reverse (antisense) primers to create templates B and C, respectively (Esposito et al., 2007). Templates A and B were then fused using a forward internal promoter primer and a reverse internal target gene primer to create the sense construct (Product D). Templates A and C were fused using the forward internal promoter primer and the forward internal target gene primer to create the antisense construct (Product E). At least 3 products were pooled and sense and antisense transgenes were microinjected at $25-100 \mathrm{ng} / \mu \mathrm{l}$, with $30 \mathrm{ng}$ of myo-3p::gfp or F25B3.3p::gfp. Multiple transgenic lines were examined for each RNAi.

All ASH-selective RNAi strains were generated by expressing RNAi under the control of $3.5 \mathrm{~kb}$ of an ASH-selective promoter (sra-6p; Troemel et al., 1995). ASI-specific RNAi strains were generated by expressing RNAi under the control of $2.8 \mathrm{~kb}$ of an ASI-specific promoter ( gpa-4p; Jansen et al., 1999). Neuron-selective RNAi transgenes were injected into either N2 or mutant animals. ADF, NSM (neurosecretory motorneuron), and AWB-specific RNAi strains were generated by expressing RNAi under the control of srh-142, ceh-2 and str-1 promoters, respectively (Troemel et al., 1997; Sagasti et al., 1999; Aspöck et al., 2003; Mukhopadhyay et al., 2007).

Generation of RNAi strains by bacterial feeding. RNA interference (RNAi) was performed as described previously using eri-1 ( $k$ 3948) animals (Kamath and Ahringer, 2003; Kennedy et al., 2004). Hermaphrodites and their progeny were grown on NGM plates containing $1 \mathrm{~mm}$ ampicillin and $1 \mathrm{~mm}$ isopropyl- $\beta$-D-thiogalactopyranosidase (IPTG) and were seeded with HT115 (DE3) bacteria containing the putative recep- 
tor RNAi vector, appropriate controls were performed. All animals were cultured at $16^{\circ} \mathrm{C}$. Synchronized second generation L4 s were picked $24 \mathrm{~h}$ preassay and examined for octanol sensitivity as described previously (Chao et al., 2004). The following RNAi animals were generated through feeding: t07d4.1RNAi, t23c6.5RNAi, r106.2RNAi, f5510.7RNAi, f42c5.2RNAi, zc84RNAi, c50f7.1RNAi, t02d1.6RNAi, y54e2a.1RNAi, y116a8b.5RNAi, k10b4.4RNAi.

Creation of rescue constructs. All rescue constructs were created by overlap fusion PCR or by cloning into pPD95.75 (Mello and Fire, 1995; Hobert, 2002). All constructs in pPD95.75 were confirmed by sequencing. (A list of strains, including primers used for the generation of rescue constructs, is available in supplemental Table S1, available at www. jneurosci.org as supplemental material.)

Localization of npr-17p::gfp. Constructs for $n p r-17:: g f p$ localization were created by an overlap of two PCR products and PCR fusion (Hobert, 2002). The strain, RWK40N2fvEx15[npr-17p::gfp] expresses a transcriptional $n p r-17:: g f p$ transgene in wild-type animals. To create the $n p r-17:: g f p$ transcriptional fusion that included $5 \mathrm{~kb}$ of promoter sequence, exon 1 and the first intron, primers NPR-17F and NPR-17:GFPtagR were used to amplify $n p r-17$. GFP was amplified from the pPD95.75 vector (GFP F + GFP R), and then fused in frame with the $n$ pr-17 product using nested primers (NPR-17 nested forward $\left(\mathrm{F}^{*}\right)$ and GFP nested reverse $\left(\mathrm{R}^{*}\right)$. Constructs were pooled from at least three reactions and coinjected with rol-6 plasmid and carrier DNA into gonads of wild-type and null mutant animals by standard techniques. Uptake of 1,1'-dioctadecyl-3,3,3',3'-tetramethylindodicarbocyanine perchlorate (DiD) in living animals was assayed as described previously (Herman and Hedgecock, 1990). Briefly, a stock solution (1 mM) of DiD (Molecular Probes/Invitrogen Labeling and Detection) was diluted 1:200 in M9 buffer. Animals were incubated in $100 \mu \mathrm{l}$ of diluted DiD for $1 \mathrm{~h}$ at RT, transferred to a fresh NGM plate seeded with OP50 and allowed to crawl on the bacterial lawn for $1-2 \mathrm{~h}$ to destain and were then placed on agarose pads with $20 \mathrm{~mm}$ sodium azide for visualization. At least three transformed lines were analyzed for $g f p$ fluorescence and DiD staining using an Olympus confocal microscope.

Creation of transgenes and strains. To create transgenic rescue or overexpressor animals, full-length or cell-specific transgenes were generated by PCR fusion as described in (Hobert, 2002). The full-length genomic $n l p-3$ transgene includes a $3 \mathrm{~kb} n l p-3$ promoter, the $n l p-3$ open reading frame and $618 \mathrm{bp}$ of the $n l p-33^{\prime}$ UTR. The full-length eat-4 genomic transgene includes a $2 \mathrm{~kb}$ eat- 4 promoter, the eat- 4 open reading frame and $1.3 \mathrm{~kb}$ of the eat- $43^{\prime} \mathrm{UTR}$. The full-length genomic egl-21 transgene includes a $1.3 \mathrm{~kb}$ egl-21 promoter, the egl-21 open reading frame and 2.2 $\mathrm{kb}$ of the egl-21 3'UTR. The full-length $n p r-17$ transgene includes a $5 \mathrm{~kb}$ promoter, the $n p r-17$ reading frame and $700 \mathrm{bp}$ of $n p r-173^{\prime} \mathrm{UTR}$. A full-length genomic ser- 5 transgene includes a $5 \mathrm{~kb}$ promoter, the ser- 5 reading frame and $1 \mathrm{~kb}$ of ser-5 3'UTR. ASH-selective transgenes express the relevant cDNAs under the control of a $3.5 \mathrm{~kb}$ ASH selective promoter (sra-6p; Troemel et al., 1995). At least 3 products were pooled and microinjected at $1-40 \mathrm{ng} / \mu \mathrm{l}$, with $30 \mathrm{ng}$ of $m y o-3 p:: g f p$ or F25B3.3p::gfp marker and at least 3 transgenic lines were examined (Kramer et al., 1990; Mello and Fire, 1995).

Derivation of phylogenetic trees. Predicted C. elegans neuropeptide receptors protein sequences were identified by BLAST and downloaded from WORMBASE as described previously for the putative C. elegans monoamine receptors (Smith et al., 2007). Each sequence was annotated to remove hypervariable regions; specifically the $\mathrm{N}$ termini were deleted 5 aa before predicted TM I, the predicted third intracellular loops 10 aa after TMV and before TMVI and the predicted C termini 15 aa after TMVII. Annotated sequences were initially aligned using the ClustalW algorithm within MegAlign (DNAStar) using default parameters (pairwise and multiple gap opening penalty of 10 , and gap extension penalties of 0.1 and 0.2 , respectively) and finalized using manual adjustment (all alignments available upon request). The statistical reliability of tree branching was assessed using bootstrap analysis (1000 replicates with random seed), and trees were compiled using the Phylogenetic Alignment Utility Program (PAUP, version 10.3). Trees were obtained using an optimality criterion of maximal parsimony and a neighbor-joining search algorithm. Accession numbers of neuropeptide G-protein-coupled receptors (GPCRs) used in the align- ment are listed in the legend to supplemental Figure 1 (available at www. jneurosci.org as supplemental material).

Multiple alignment of NPR-17 with closely related peptide receptors (supplemental Fig. 2, available at www.jneurosci.org as supplemental material). Related neuropeptide receptors protein sequences were identified by BLAST as described previously for the putative C. elegans monoamine receptors (Smith et al., 2007). Each sequence was annotated to remove hypervariable regions; specifically the $\mathrm{N}$ termini were deleted 5 aa before predicted TM I, the predicted third intracellular loops 10 aa after TMV and before TMVI and the predicted C termini 15 aa after TMVII. Annotated sequences were initially aligned using MegAlign in DNAStar with ClustalW using default parameters and fine tuned by hand.

\section{Results}

\section{Both $\mathrm{G} \boldsymbol{\alpha}_{\mathrm{q}}(\mathrm{EGL}-30)$ and $\mathrm{G} \boldsymbol{\alpha}_{\mathrm{s}}(\mathrm{GSA}-1)$ signaling in the ASH} sensory neurons increase aversive responses to dilute octanol

The ASH sensory neurons are necessary and sufficient for aversive responses to dilute octanol and the expression of the GPCR, ser-5, in the ASHs is essential for food or 5-HT stimulation of the aversive response (Chao et al., 2004; Harris et al., 2009). For example, ser-5-null animals do not increase aversive responses in the presence of food or 5-HT, in contrast to wild-type animals, and 5-HT stimulation can be restored by the expression of ser-5 in the ASHs (Fig. 1) (Harris et al., 2009). Conversely, wild-type animals overexpressing ser-5 exhibit more rapid responses off food (Fig. 1). To characterize the signaling pathways involved in the serotonin receptor-5 (SER-5)-mediated modulation of the ASHs, individual G-protein signaling mutants were examined for octanol avoidance, including if mutants for a PDE4 cAMP phosphodiesterase ( $p d e-4$; Charlie et al., 2006) and gain-of function mutants for $\mathrm{G} \alpha_{\mathrm{s}}$ ( $g s a-1 g f$; Schade et al., 2005), G $\alpha_{\mathrm{q}}$ (egl-30gf; Hawasli et al., 2004) and adenylyl cyclase (acy-1gf; Schade et al., 2005). Animals with increased $\mathrm{G} \alpha_{\mathrm{q}}(e g l-30 g f)$ or $\mathrm{G} \alpha_{\mathrm{s}}$ ( $g s a-1 g f$, $p d e-4)$ signaling exhibited more rapid responses to dilute octanol off food than wild-type animals that mimicked responses observed in the presence of food or 5-HT, suggesting that both signaling pathways might be involved in the food/5-HT sensitization of the ASHs (Fig. 1; see diagram, Fig. 12).

To confirm that these G-protein signaling pathways were stimulating the ASHs directly, ASH-selective RNAi was used to specifically interfere with G-protein signaling (Esposito et al., 2007). This RNAi approach is reported to yield efficient, heritable, knockdown that does not spread significantly to other neurons, at least in the case of the ASHs (Esposito et al., 2007). We have confirmed these observations using the ASH-selective promoter, sra-6p. For example, the ASH knockdown of ser-5 or octr- 1 abolished the 5-HT stimulation and OA inhibition of aversive responses, respectively, but had no effect on responses off food, suggesting that the octanol-sensing machinery in the ASHs was intact (Fig. 2). Since the sra-6 promoter also has limited expression in the ASI sensory neurons, an ASI-specific promoter $(g p a-4 p)$ was used as a control to knockdown many of the genes examined in the present study. As predicted, the ASI RNAi knockdown of ser-5 or octr- 1 had no effect on aversive responses, confirming that our results were based on knockdown in the ASHs and also that spreading of the RNAi is limited (Fig. 2).

The ASH-selective RNAi knockdown of $\mathrm{G} \alpha_{\mathrm{q}}$ (egl-30) decreased aversive responses off food and the knockdown of either $\mathrm{G} \alpha_{\mathrm{q}}$ or $\mathrm{G} \alpha_{\mathrm{s}}$ ( gsa-1) abolished 5-HT stimulation (Fig. 2). In contrast, the ASH RNAi knockdown of either pde-4, a 5' phosphodiesterase (Charlie et al., 2006) or kin-2, a regulatory subunit of the cAMP-dependent protein kinase (Lu et al., 1990), increased aversive responses off food to levels similar to those observed with 5 -HT. Similarly the ASH RNAi knockdown of $d g k-1$, a diacylglyc- 
erol (DAG) kinase also increased aversive responses off food to levels similar to those observed with 5-HT, presumably by increasing DAG levels downstream of ASH G $\alpha_{\mathrm{q}}$ signaling (Miller et al., 1999; Nurrish et al., 1999). Together these results suggest that that activating either $\mathrm{G} \alpha_{\mathrm{s}}$ or $\mathrm{G} \alpha_{\mathrm{q}}$ signaling sensitized the ASHs (Fig. 2).

To characterize the role of SER-5 in ASH sensitization, we attempted to increase aversive responses in ser-5-null animals by activating individual G-protein-mediated pathways. Both egl-30gf ser-5 and egl-30gf animals exhibited increased responses compared with ser-5 animals (Figs. 1, 3). Similarly, the ASH RNAi knockdown of $d g k-1$ in either wild-type or ser-5 animals also increased aversive responses both off food and in the presence of 5-HT (Figs. 2, 3 ). Together, these results suggest that $\mathrm{G} \alpha_{\mathrm{q}}$ (EGL-30)functions downstream or in parallel to ser-5; however, whether SER- 5 couples directly to $\mathrm{G} \alpha$ and stimulates neurotransmitter release or acts upstream to modulate neuronal excitability remains to be determined. In contrast, gsa-1gf ser-5, ser-5;pde-4 or ser- 5 animals with pde-4 knocked down in the ASH by RNAi did not exhibit the elevated aversive responses observed by activating $\mathrm{G} \alpha_{\mathrm{s}}$ signaling in the ASHs of wild-type animals (Fig. 3). These results suggest that SER-5-dependent $\mathrm{G} \alpha_{\mathrm{q}}$ activation is essential for $\mathrm{G} \alpha_{\mathrm{s}}$-stimulated increased responses off food.

Octopamine inhibits aversive responses by activating OCTR-1 and $\mathrm{G} \alpha_{\mathrm{o}}$ in the ASHs

OA inhibits food or 5-HT stimulated aversive responses to dilute octanol through the OA receptor, OCTR-1, expressed in the ASHs (Wragg et al., 2007) (Fig. 1). For example, 5-HT stimulated aversive responses were not inhibited by OA in octr1-null animals or in wild-type animals with octr-1 knocked down in the ASHs by RNAi (Figs. 1, 2) (Wragg et al., 2007). OA also inhibited the increased aversive responses observed off food in animals with elevated cAMP levels ( $g s a-1 g f$, acy-1gf, pde-4) (Fig. 1). Similarly, OA inhibited the elevated responses off food observed after the ASH RNAi knockdown of pde-4 (Fig. 2). In contrast, the ASH RNAi knockdown of $\mathrm{G} \alpha_{\mathrm{o}}$ ( goa-1) abolished the OA inhibition of 5-HT stimulation, but had no effect on responses off food or in the presence of 5-HT, suggesting that OA and OCTR-1 signal through G $\alpha_{\mathrm{o}}$ in the ASHs to inhibit 5-HT stimulation (Fig. 2). Presumably, the absence of elevated signaling off food after $\mathrm{G} \alpha_{\mathrm{o}}$ knockdown reflects a paucity of endogenous OA and therefore, limited $\mathrm{G} \alpha_{\mathrm{o}}$ activation.
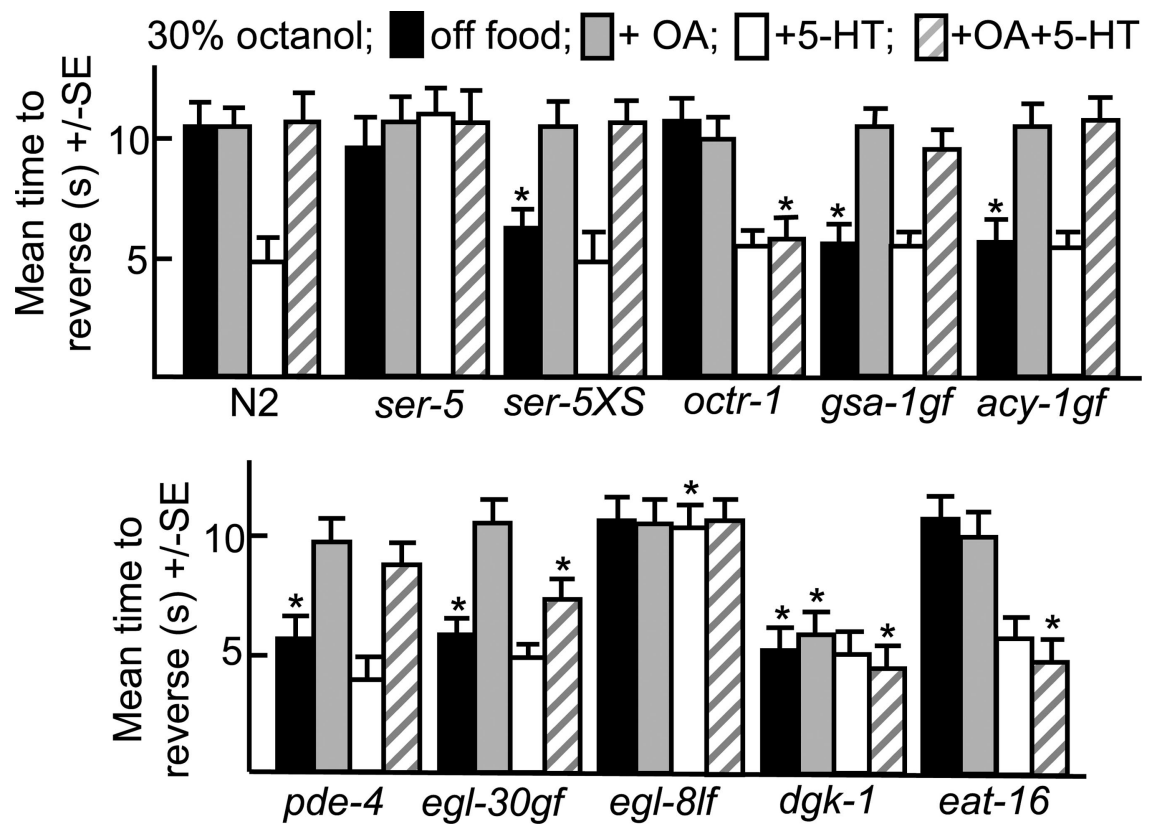

Figure 1. Monoamine-mediated G-protein signaling modulates ASH-mediated aversive responses to dilute octanol. Wild-type and G-protein signaling mutants were examined for aversive responses to dilute (30\%) octanol in the presence and absence of exogenous 5-HT, OA or 5-HT and OA ( 4 mM), as described in Materials and Methods. Data are presented as mean \pm SE and analyzed by two-tailed Student's $t$ test. ${ }^{*} p<0.001$, significantly different from wild-type animals under identical test conditions. Black, Off food; gray, +OA; white, +5-HT; hatched, +5-HT+OA.

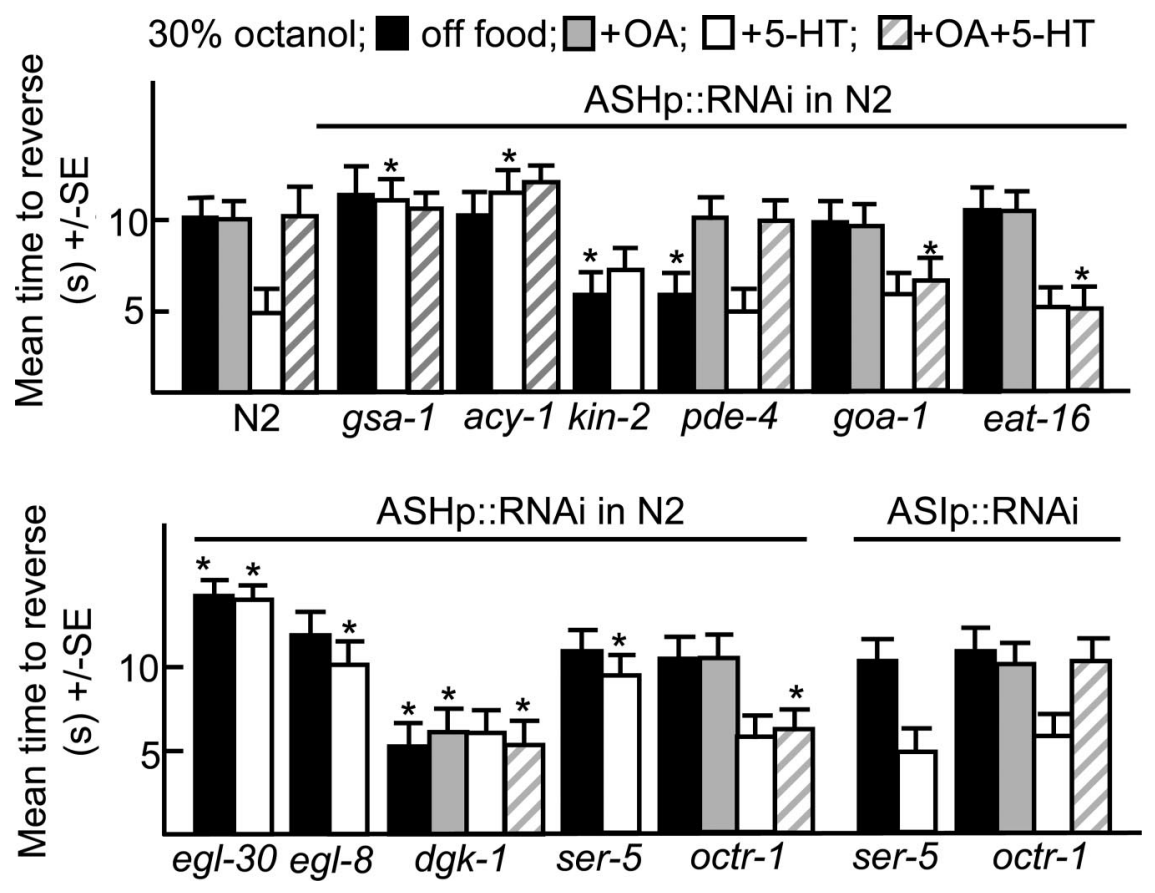

Figure 2. Monoamines modulate G-protein signaling in the ASH sensory neurons. Wild-type animals expressing RNAi transgenes were assayed for aversive responses to dilute (30\%) octanol in the presence or absence of exogenous 5-HT, 0A, or 5-HT and $+O A(4 \mathrm{~mm})$, as described in Materials and Methods. Data are presented as mean \pm SE and analyzed by two-tailed Student's t test. ${ }^{*} p<0.001$, significantly different from wild-type animals under identical test conditions. Black, Off food; gray, $+0 \mathrm{~A}$; white, +5-HT; hatched, +5-HT+0A. ASH-selective promoter, sra-6p; ASI-selective promoter, gpa-4p.

OA also inhibited increased responses off food in $\mathrm{G} \alpha_{\mathrm{q}}$ (egl$30 g f$ ) animals and this OA inhibition appeared to involve signaling in the ASHs, as the OA inhibition of egl-30gf animals was abolished by the ASH RNAi knockdown of octr-1 (Fig. 1; see Fig. 5). G $\alpha_{\mathrm{o}}$ may decrease signaling in the ASHs by inhibiting adenylyl 


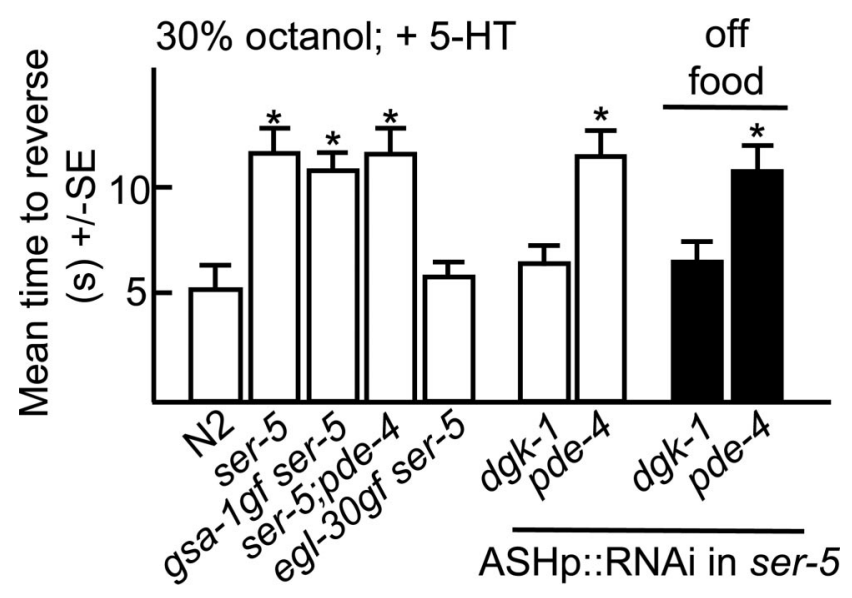

Figure 3. SER-5 signaling in ASH sensory neurons. Wild-type and mutant animals were examined for aversive responses to dilute $(30 \%)$ octanol either in the presence or absence of exogenous 5-HT (4 mM), as described in Materials and Methods. Data are presented as mean \pm SE and analyzed by two-tailed Student's $t$ test. ${ }^{*} p<0.001$, significantly different from wildtype animals under identical test conditions. ASH-selective promoter, sra-6p.

cyclase. Alternatively, since $\mathrm{G} \alpha_{\mathrm{o}}$ (GOA-1) inhibits $\mathrm{G} \alpha_{\mathrm{q}}$ signaling, the ability of OCTR-1/G $\alpha_{\mathrm{o}}$ signaling to abolish the $\mathrm{G} \alpha_{\mathrm{s}}$ signaling responses is consistent with our finding that the increased offfood aversive response in mutants with an activated $\mathrm{G} \alpha_{s}$ pathway requires the SER-5 receptor and $\mathrm{G} \alpha_{\mathrm{q}}$ (Hajdu-Cronin et al., 1999; Miller et al., 1999) (Figs. 2, 3). EAT-16 encodes an inhibitory regulator of G-protein signaling (RGS) protein that converts $\mathrm{G} \alpha_{\mathrm{q}}$ from an active GTP-bound to an inactive GDP-bound form and If mutations in eat-16 animals were identified in a screen for suppressors of activated $\mathrm{G} \alpha_{\mathrm{o}}$ (Hajdu-Cronin et al., 1999). Interestingly, OA did not inhibit the 5-HT stimulation of aversive responses in eat-16-null animals or in animals with eat-16 knocked down in the ASHs by RNAi (Figs. 1, 2). The ASH RNAi knockdown of eat-16 had no effect on aversive responses off food or in the presence of 5-HT, as also observed after the ASH RNAi knockdown of $\mathrm{G} \alpha_{\mathrm{o}}$ (Fig. 2). In contrast, OA did not inhibit the increased responses off food observed after the ASH RNAi knockdown of $d g k-1$ (Fig. 2).

These studies suggest that OA and OCTR-1 signal through $\mathrm{G} \alpha_{\mathrm{o}}(\mathrm{GOA}-1)$ in the ASHs and may inhibit the 5-HT stimulation of aversive responses by potentially turning off SER-5/G $\alpha_{\mathrm{q}}$ signaling, which our earlier data show is necessary for the $\mathrm{G} \alpha_{\mathrm{s}}$ response. Interestingly, the OA-mediated inhibition of $\mathrm{G} \alpha_{\mathrm{q}}$ and $\mathrm{G} \alpha_{\mathrm{s}}$ driven ASH signaling appears to be sufficient to abolish food or 5-HT-dependent increases in aversive responses.

Peptides from the ASHs are required for the 5-HT stimulation of aversive responses

As noted above, ASH G $\alpha_{\mathrm{s}}$ signaling was essential for the 5-HT stimulation of aversive responses. To determine whether $\mathrm{G} \alpha_{\mathrm{s}}$ signaling stimulated DCV/neuropeptide release, the potential role of neuropeptides in the aminergic modulation of ASHmediated aversive responses was examined in egl-3 and egl-21 animals that lack a proprotein convertase and a carboxypeptidase, respectively, that are required for efficient neuropeptide processing (Fig. 4). Both egl-3 and egl-21 animals exhibit severely deficient peptide profiles (Husson et al., 2006, 2007). Indeed, both egl-3 and egl-21 animals failed to increase aversive responses in the presence of 5-HT, suggesting that peptides are essential for 5-HT stimulation. In contrast, egl-3 and egl-21 animals off food exhibited wild-type responses to dilute octanol, suggesting that

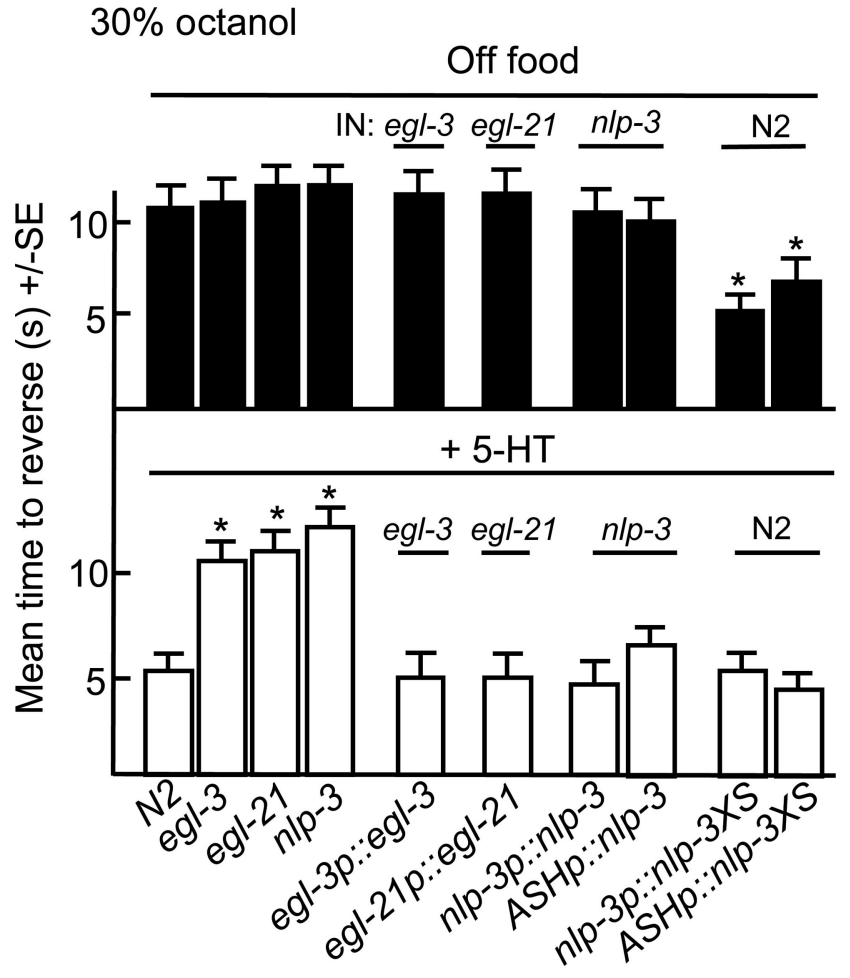

Figure 4. Peptides encoded by $n / p-3$ are essential for the 5-HT-dependent stimulation of aversive responses to dilute octanol. Wild-type, mutant, and transgenic animals were examined for aversive responses to dilute (30\%) octanol in the absence (top) or presence (bottom) of exogenous 5-HT (4 mM), as described in Materials and Methods. Data are presented as mean \pm SE and analyzed by two-tailed Student's $t$ test. ${ }^{*} p<0.001$, significantly different from wildtype animals under identical test conditions. ASH-selective promoter, sra-6p.

their octanol-sensing machinery was intact. Similar phenotypes were observed after the ASH RNAi knockdown of egl-3, confirming a role for egl-3 in the ASHs (Fig. 5).

The ASHs express at least three neuropeptide genes ( $n l p-3$, $n l p-15$ and $f l p-21)$ that encode at least nine predicted peptides (Nathoo et al., 2001). In the presence of exogenous 5-HT, aversive responses to dilute octanol in $n l p-15$ - and flp-21-null animals were identical to those of wild-type animals (data not shown). In contrast, aversive responses were not stimulated by food or 5-HT in $n l p$-3-null animals (Figs. 4-6). Conversely, the overexpression of $n l p-3$ in wild-type animals from either the native $n l p-3$ or ASH-selective promoters increased aversive responses off food (Fig. 4). $n l p-3$ is expressed in many sensory neurons, including the ADFs, ASEs, ASHs, ASJs, and AWBs (Nathoo et al., 2001). However, 5 -HT stimulation was fully rescued in $n l p-3$ animals by the ASH-selective expression of $n l p-3$ and the ASH-selective RNAi knockdown of $n l p-3$ abolished food or 5-HT stimulation to levels observed in the $n l p-3$-null animals (Figs. 4-6). Together, these results suggest that the release of peptides encoded by $n l p-3$ from the ASHs is sufficient to stimulate aversive responses to dilute octanol.

G $\boldsymbol{\alpha}_{\mathrm{s}}$ (GSA-1) signaling in the ASHs appears to stimulate the release of peptides encoded by $n l p-3$

The ASH RNAi knockdown of egl-3, in $g s a-1 g f$ animals abolished the increased aversive responses observed in these $g$ f mutants off food, suggesting the $\mathrm{G} \alpha_{\mathrm{s}}$ signaling was stimulating peptide release (Fig. 5). Therefore, to identify the G-protein signaling pathways specifically modulating the release of $n l p$-3-encoded peptides from the ASHs, additional mutants were created. In agreement 
with the egl-3 data, gsa-1gf;nlp-3 and $p d e-$ 4;nlp-3 animals did not exhibit the elevated responses off food observed in gsa-1gf or pde-4 animals (Fig. 5). Similarly, the ASH RNAi knockdown of pde-4 in nlp-3-null animals also failed to increase responses off food, in contrast to the ASH RNAi knockdown of $p d e-4$ in wild-type animals (Figs. 2, 5). In contrast, both egl-30gf and egl-30gf;nlp-3 animals exhibited elevated responses off food (Figs. 1, 5). Similarly, the ASH RNAi knockdown of $d g k-1$ in either wild-type, egl-3 or $n l p-3$ animals also stimulated responses off food, suggesting the $n l p-3$ was not downstream of $\mathrm{G} \alpha_{\mathrm{q}}$ and that $\mathrm{G} \alpha_{\mathrm{q}}$ stimulated increases in glutamate alone might be sufficient to increase ASHmediated signaling (Figs. 2, 5).

Together, these results suggest that ASH-mediated aversive responses to dilute octanol can be increased by either (1) activating $\mathrm{G} \alpha$ q signaling by the overexpression or activation of SER-5/EGL-30 or the inhibition of diacylglycerol kinase- 1 or (2) activating $\mathrm{G} \alpha_{s}$ signaling and peptide release through the activation GSA-1/ACY-1, the inhibition of PDE- 4 or the overexpression of peptides encoded by $n l p-3$.

\section{Serotonin stimulates aversive responses when glutamatergic signaling in the ASHs is compromised through a pathway requiring peptides encoded by $n l p-3$}

To confirm the stimulatory role of $n l p-3$-encoded peptides on ASH-mediated aversive responses, eat-4-null animals that lack a vesicular glutamate transporter essential for glutamatergic transmission were examined (Lee et al., 1999). Not surprisingly, eat-4 animals or wild-type animals with eat-4 knocked down by RNAi in the glutamatergic ASHs responded very poorly $(>18$ and $16 \mathrm{~s}$, respectively), if at all, to dilute octanol, since these assays were terminated after $20 \mathrm{~s}$ to minimize complications introduced by spontaneous reversal (Fig. 7). However, aversive responses in eat -4 animals or animals with eat- 4 knocked down in the ASHs by RNAi were still dramatically stimulated by 5 -HT (Fig. 7). In contrast, aversive responses in eat-4;egl-3, eat-4;nlp-3 animals, or eat-4-null animals with $n l p-3$ knocked down in the ASHs by RNAi were not stimulated by 5 -HT, suggesting that 5 -HT was stimulating aversive responses by stimulating the release of $n l p-$ 3 -encoded peptides in the eat-4 animals (Fig. 7). This apparent 5-HT stimulation of peptide release was unanticipated, given that SER-5 appears to signal through a pathway requiring $\mathrm{G} \alpha_{\mathrm{q}}$ and peptide release appears to be dependent on $\mathrm{G} \alpha_{\mathrm{s}}$ (Figs. 1, 2). Interestingly, the knockdown of either $\mathrm{G} \alpha \alpha_{\mathrm{s}}, \mathrm{G} \alpha_{\mathrm{q}}$ or UNC-13 in eat-4 animals abolished 5-HT stimulation (Fig. 7).

Together, these results demonstrate that (1) in the absence of the ASH EAT-4 vesicular glutamate transporter, animals respond very poorly to dilute octanol, supporting a role for EAT- 4 as the major ASH glutamate transporter, (2) aversive responses in eat-4 animals are still stimulated by 5 -HT, while aversive responses in eat-4;nlp-3 or eat-4 animals with $n l p-3$ knocked down in the ASHs are not, further supporting a role for $n l p-3$ in 5-HT stimulation and (3) in the absence of significant glutamatergic signaling both ASH $\mathrm{G} \alpha_{\mathrm{s}}$ and $\mathrm{G} \alpha_{\mathrm{q}}$ signaling are essential for 5 -HT stimulation.

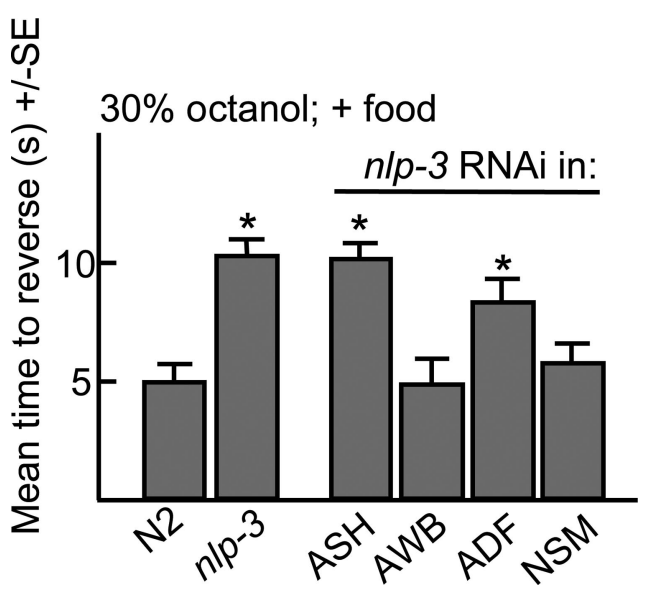

Figure 6. The RNAi knockdown of $n / p-3$ in the ASHs abolishes the food stimulation of aversive responses to dilute octanol. Aversive responses to dilute octanol were examined in wildtype animals in the presence of $E$. coli (OP50) after the promoter-selective RNAi knockdown of nlp-3 (ASHs, sra-6p::nlp-3; NSMs, ceh-2p::nlp-3; ADFs, srh-142p::nlp-3; AWBs, str-1p::nlp-3). Data are presented as mean \pm SE and analyzed by two-tailed Student's $t$ test. ${ }^{*} p<0.001$, significantly different from wild-type animals under identical test conditions. Gray, +Food.

\section{Peptides encoded by $n l p-3$ activate NPR-17 to increase} aversive responses

G-protein coupled receptors (GPCRs) potentially activated by $n l p-3$-encoded peptides were identified by screening predicted peptide receptor-null mutants for phenotypes identical to those observed in $n l p-3$ animals. The C. elegans genome contains $>50$ genes that encode putative peptide GPCRs and some of these genes have been identified previously based on potential homology to mammalian peptide receptors. For example, putative NPY-like receptors have been identified using this approach (Cohen et al., 2009). We used a bioinformatics approach to identify predicted neuropeptide receptors (supplemental Fig. S1, available at www.jneurosci.org as supplemental material). This analysis used predicted sequences from truncated receptors lacking potential hypervariable regions, including most of the predicted $\mathrm{N}$ and $\mathrm{C}$ termini and third intracellular loops; an approach used previously to identify C. elegans monoamine receptors (Smith et 


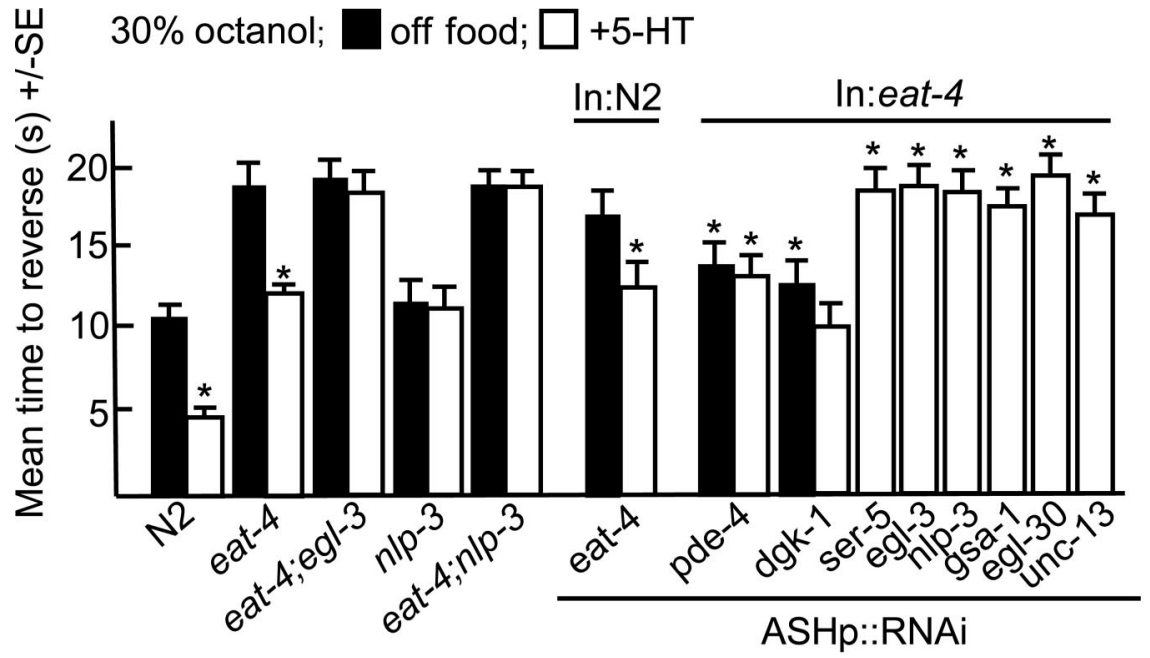

Figure 7. 5-HT stimulates ASH-mediated aversive responses in eat-4-null animals. Wild-type and mutant animals were examined for aversive responses to dilute octanol (30\%) in the presence or absence of exogenous 5 -HT ( $4 \mathrm{mM})$, as described in Materials and Methods. Data are presented as mean \pm SE and analyzed by two-tailed Student's $t$ test. ${ }^{*} p<0.001$, significantly different from animals incubated in the absence of 5 -HT. ${ }^{*}$ Significantly different from eat-4-null animals incubated under identical conditions. Black, Off food; white, +5-HT. ASH-selective promoter, sra-6p.

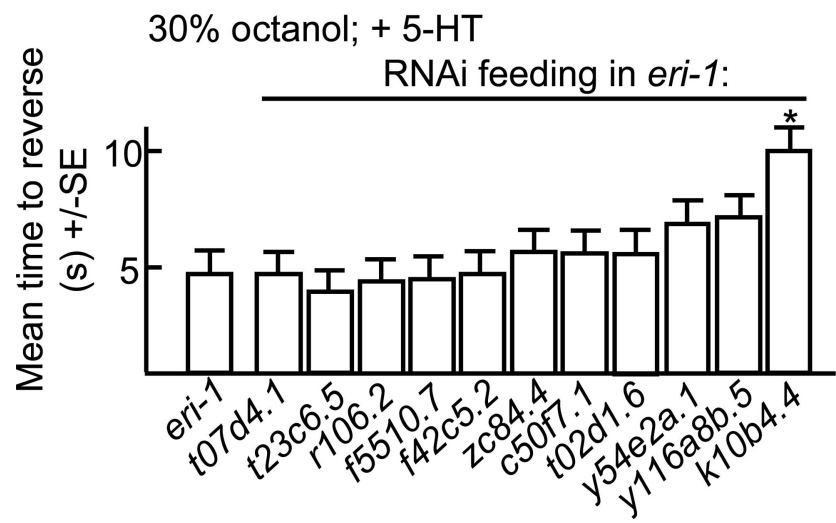

Figure 8. 0ctanol avoidance in eri-1 animals after the knockdown of predicted neuropeptide receptors by RNAi feeding. eri-1 (kp3948) animals expressing global RNAi after bacterial feeding were examined for aversive responses to dilute octanol in the presence of 5-HT ( $4 \mathrm{mM})$. Data are presented as mean \pm SE and analyzed by two-tailed Student's $t$ test. ${ }^{*} p<0.001$, significantly different from wild-type animals under identical conditions.

al., 2007). Indeed, most of the NPY-like receptors identified previously clustered using this approach (NPR-1-14; supplemental Fig. S1, available at www.jneurosci.org as supplemental material). Initially, animals with mutations in non-NPY-like receptors were assayed for 5-HT-dependent increases in aversive responses. Putative null alleles were available for many of these receptors; others were examined using RNAi in an eri-1-null background that is more sensitive to neuronal RNAi knockdown (Kennedy et al., 2004) (Fig. 8). eri-1 animals exhibited wild-type responses to dilute octanol (Fig. 8). Eight predicted receptor-null mutants that did not increase aversive responses to dilute octanol in the presence of 5-HT were identified using this approach (npr10, npr-17, t02e9.1, t02e9.3, t11f9.1, f02e8.2, t07d10.2, and k10b4.4; Figs. 8, 9). This relatively large number of receptors might be expected, as $n l p-3$ encodes multiple peptides that may activate distinct receptors. For example, peptides encoded by flp-18 activate multiple GPCRs (Cohen et al., 2009). In addition, we have identified other peptideencoding genes that contribute to 5-HT sensitization (data not shown). To identify the specific GPCR(s) activated by $n l p-3$ peptides, $n l p-3$ was overexpressed in the receptor-null animals that were not stimulated by 5 -HT. Of these seven mutants, only npr-17(c06g4.5)-null animals failed to increase aversive responses off food after $n l p-3$ overexpression (Fig. 9). As predicted, the expression of $n p r-17$ (npr-17p::npr-17) in $n p r-17$-null animals rescued 5-HT stimulation (Fig. 9). In addition, the overexpression of $n p r-17$ in wild-type animals increased aversive responses off food, as observed above for $n l p-3$ overexpression, and this increased response off food was absent when $n p r-17$ was overexpressed in $n l p$-3-null animals (Fig. 9). NPR-17 is most closely related to a predicted Brugia malayi ORL1-like opioid receptor (68\% identity; E value $4 \times$ $10^{-138}$ ) and two other C. elegans predicted peptide receptors, C43C3.2 (34\%; $4 \times 10^{-62}$ ) and T02D1.6 (37\%; $3 \times$ $10^{-59}$; supplemental Fig. S2, available at www.jneurosci.org as supplemental material). In addition, NPR-17 exhibits significant identity to a mammalian somatostatinlike receptor $\left(28 \% ; 6 \times 10^{-21}\right)$ and melanocyte-concentrating hormone receptor $1\left(26 \% ; 2 \times 10^{-17}\right.$, supplemental Fig. S2, available at www.jneurosci.org as supplemental material). $n p r-17$ appears to be expressed in the AVG, faintly in the ASIs and at least one additional pair of unidentified neurons in the head and the PVPs, PVQs, and $\mathrm{PQR}$ in the tail, based on fluorescence from an $n p r-17 p:: g f p$ transgene (Fig. 10).

To confirm that NLP-3 (neuropeptide-like) signaling was mediated by NPR-17, other $n l p-3$-null phenotypes were examined in the $n p r-17$-null animals. For example, both $n l p-3$ - and $n p r-17-$ null animals spontaneously reversed significantly less frequently immediately off food than wild-type animals (Fig. 11). Similarly, both nlp-3- and npr-17-null animals were hypersensitive to the OA inhibition of aversive responses to $100 \%$ octanol and the expression of $n l p-3$ abolished OA inhibition in wild-type, but not npr-17-null animals (Fig. 11). Together, these results suggest that NPR-17 is activated by $n l p-3$-encoded peptides.

\section{Discussion}

Behavioral state in C. elegans is dependent on food availability and is defined by the selective release of both monoamines and peptides. For example, 5-HT/OA ratios define nutritional status and antagonistically modulate most key behaviors (Horvitz et al., 1982; Alkema et al., 2005; Wragg et al., 2007). The present studies demonstrate that $5-\mathrm{HT}$ and $\mathrm{OA}$ also antagonistically modulate aversive behaviors mediated by the ASHs and that release of $n l p-3$ peptides from the ASHs is essential for the 5-HT stimulation of aversive responses. Both $\mathrm{G} \alpha_{\mathrm{q}}$ and $\mathrm{G} \alpha_{\mathrm{s}}$ signaling in the ASHs are essential for 5-HT sensitization, with $\mathrm{G} \alpha_{\mathrm{s}}$ signaling specifically stimulating neuropeptide release (Fig. 12). The role of G-protein signaling in modulating aversive responses in the ASHs is consistent with that predicted for neurotransmitter release from $C$. elegans motorneurons (Perez-Mansilla and Nurrish, 2009). For example, the $\mathrm{G} \alpha_{\mathrm{s}}$ pathway also exerts its effects on locomotion in a $\mathrm{G} \alpha_{\mathrm{q}}$ pathway-dependent manner (Reynolds et al., 2005). How- 
A

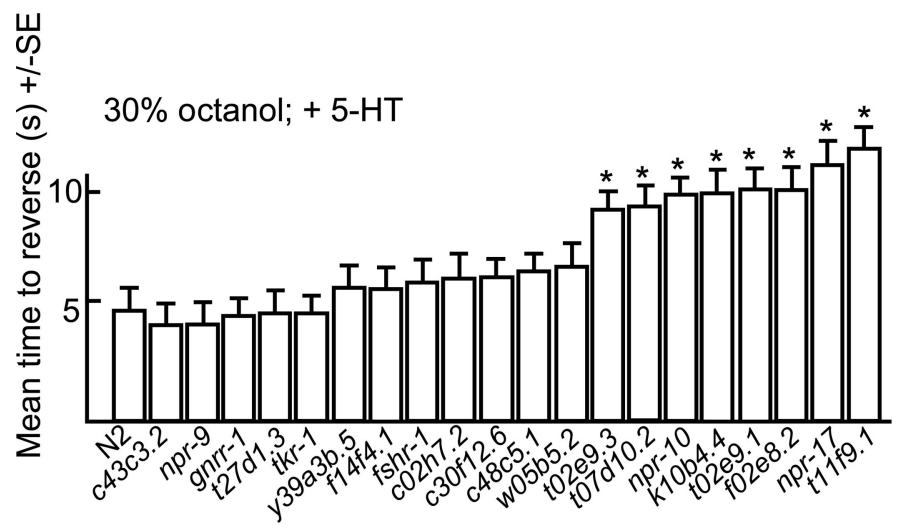

B

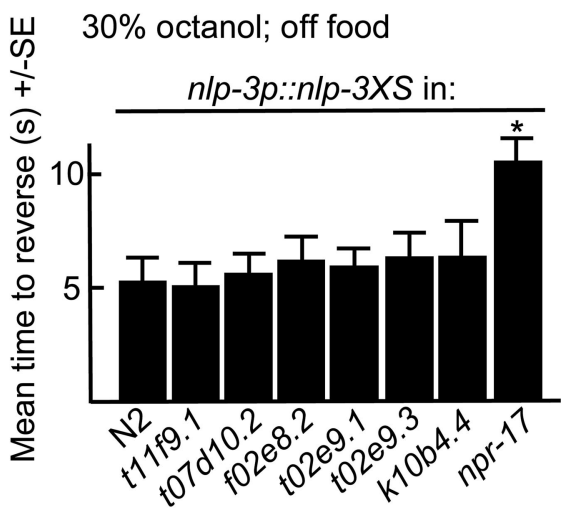

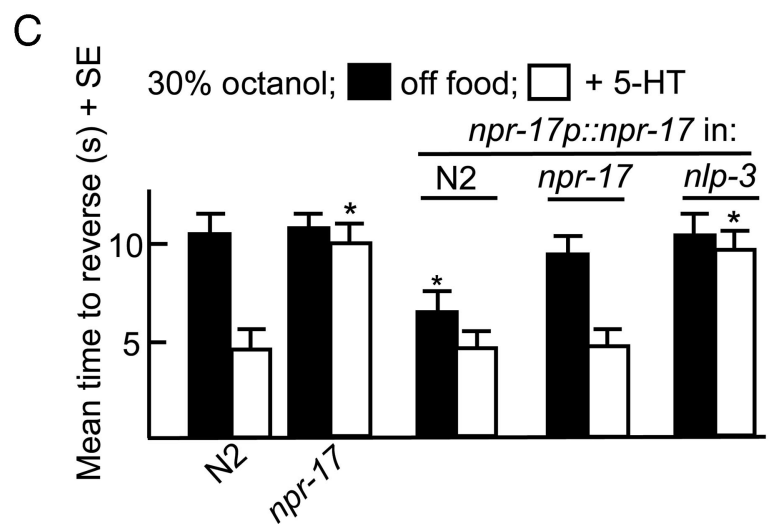

Figure 9. $\quad n p r-17$ encodes a neuropeptide receptor that appears to be activated by $n / p$-3-encoded peptides. $A$, Putative neuropeptide receptor-null mutants were examined for 5 -HT-dependent increases in aversive responses to dilute octanol (30\%), as described in Materials and Methods. B, Putative neuropeptide receptor-null mutants overexpressing nlp-3p::nlp-3 were examined for aversive responses to dilute octanol. C, Wild-type and npr-17-null animals expressing npr-17p::npr-17 transgenes were examined for aversive responses to dilute octanol in the presence or absence of 5-HT. Black, Off food; white, +5 -HT. Data are presented as mean \pm SE and analyzed by two-tailed Student's $t$ test. ${ }^{*} p<0.001$, significantly different from wild-type animals under identical test conditions.
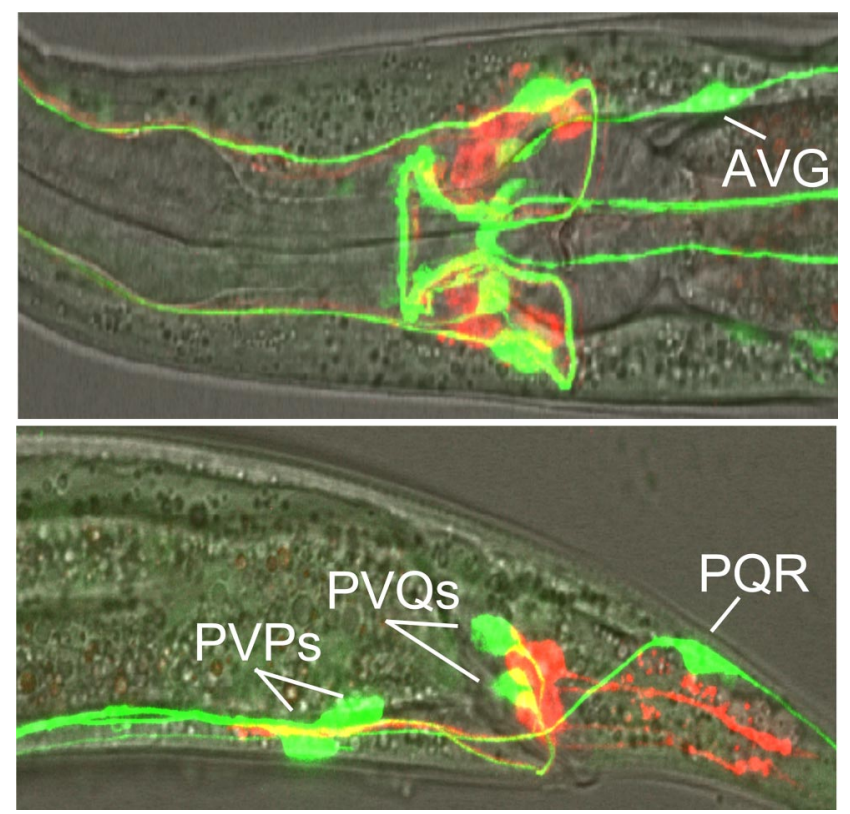

Figure 10. $n p r-17$ is expressed in subsets of head and tail neurons. GFP fluorescence from a npr-17p::gfp transcriptional fusion. $n p r-17$ transgene includes 5 kb upstream of the start ATG, as well as the first exon and intron of npr-17, fused to sequence encoding GFP. Merge of GFP fluorescence and DiD (a lipophilic dye) staining in the nerve ring.

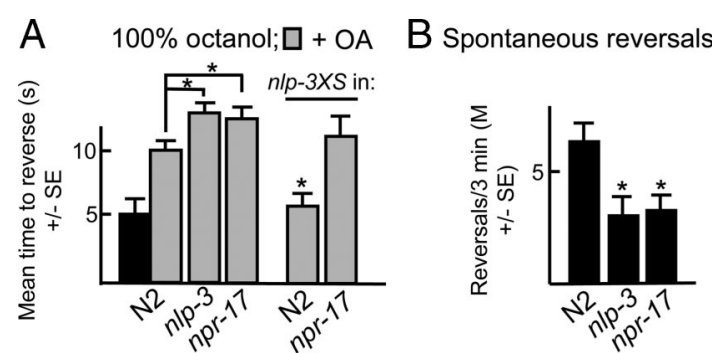

Figure 11. n/p-3-and npr-17-null animals exhibit identical phenotypes. Wild-type and mutant animals were examined for aversive responses to $100 \%$ octanol in the presence and absence of $\mathrm{OA}(4 \mathrm{~mm})$ and spontaneous reversal immediately off food. Data are presented as mean \pm SE and analyzed by two-tailed Student's $t$ test. ${ }^{*} p<0.001$, significantly different from wild-type animals under identical test conditions.

ever, whether these G-protein signaling cascades directly modulate glutamate and/or neuropeptide release remains to be determined. For example, ASH signaling requires the activity of an OSM-9/OCR-2 TRPV ion channel in the sensory cilia and in other systems $\mathrm{G} \alpha$, signaling is involved in TRPV activation (for review, see Colbert et al., 1997; Montel, 2005; Bergamsco and Bazzicalupo, 2006). Similarly, gap junctions are also modulated by monoaminergic signaling (Rorig and Sutor, 1996; Bloomfield and Völgyi, 2009; Kothmann et al., 2009). The ASHs offer advantages over motorneurons for studies of G-protein signaling and behavioral plasticity. For example, ASH postsynaptic partners are 


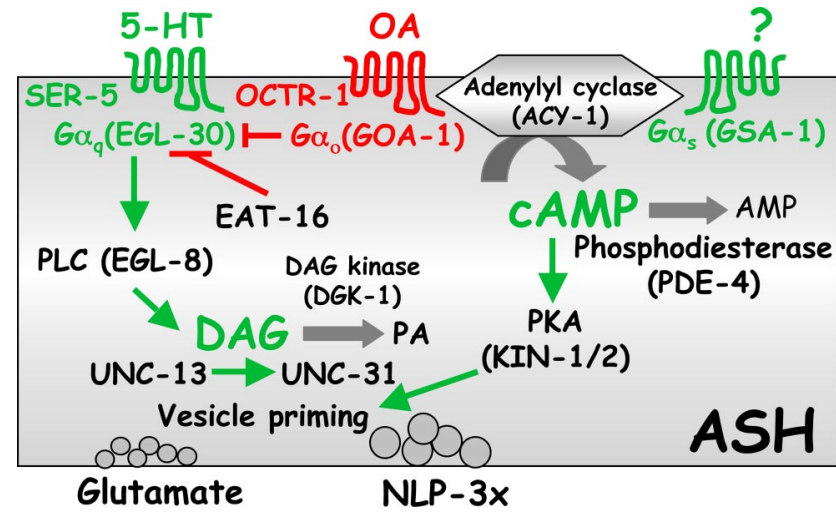

Figure 12. Model of G-protein modulation of aversive responses mediated by the ASH sensory neurons. Green and red represent stimulation and/or inhibition, respectively, of aversive responses to dilute octanol.

neurons, not muscles, $\mathrm{Ca}^{2+}$ dynamics are modulated directly by sensory cues (White et al., 1986; Hilliard et al., 2005), modulatory peptides and monoamine/peptide receptors have been identified, and the ASHs are amenable to RNAi permitting the selective manipulation of ASH signaling directly. In contrast, we have observed off target effects using this RNAi approach in cholinergic motorneurons, probably, in part, because of the large number of motorneurons expressing the dsRNAs.

\section{G-protein signaling in the ASHs modulates} aversive responses

Activating either $\mathrm{G} \alpha_{\mathrm{q}}$ or $\mathrm{G} \alpha_{\mathrm{s}}$ signaling in the ASHs increases aversive responses. In contrast, inhibition of either $\mathrm{G} \alpha$, or $\mathrm{G} \alpha$ s signaling in the ASHs abolishes 5-HT stimulation. These results suggest that 5-HT stimulation requires both $\mathrm{G} \alpha_{\mathrm{q}}$ and $\mathrm{G} \alpha_{\mathrm{s}}$ signaling, but that the activation of either pathway is sufficient to increase aversive responses, as long as basal levels of signaling in the other pathway are maintained. The receptor activating $\mathrm{G} \alpha$ in the ASHs has not been identified, but does not appear to be SER-5. For example, gsa-1gf ser-5 or ser-5;pde-4 mutants or ser-5 animals with $p d e-4$ knocked-down in the ASHs are not stimulated by 5-HT and do not exhibit the elevated responses off food observed in $g s a-1 g$ for $p d e-4$ animals, suggesting that $\mathrm{G} \alpha_{\mathrm{s}}$ does not function downstream of SER-5. In contrast, the ASH RNAi knockdown of ser-5 has no effect on the increased aversive responses off food associated with increased $\mathrm{G} \alpha_{\mathrm{q}}$ signaling, suggesting that $\mathrm{G} \alpha_{\mathrm{q}}$ functions downstream of SER-5; whether SER-5 couples directly to $\mathrm{G} \alpha$ q remains to be determined.

OA inhibits the food/5-HT stimulation of aversive responses through OCTR-1 (F14D12.6) expressed in the ASHs (Wragg et al., 2007). OA also inhibits increased responses off food associated with increased $\mathrm{G} \alpha_{\mathrm{s}}$ signaling and the ASH RNAi knockdown of either octr-1 or $\mathrm{G} \alpha_{\mathrm{o}}$ abolishes the OA inhibition of food/5-HTdependent stimulation, suggesting that OCTR-1 activates $\mathrm{G} \alpha_{\mathrm{o}}$. $\mathrm{OA}$ and $\mathrm{G} \alpha_{\mathrm{o}}$ may inhibit ASH signaling by negatively regulating adenylyl cyclase and/or inhibiting $\mathrm{G} \alpha$, perhaps through the RGS protein, EAT-16, as OA did not inhibit the 5-HT stimulation of aversive responses in eat-16-null animals or animals with eat-16 knocked down in the ASHs. Clearly, $\mathrm{G} \alpha_{\mathrm{o}}$ inhibits $\mathrm{G} \alpha$, signaling in C. elegans, but the specifics of $\mathrm{G} \alpha_{\mathrm{o}}$ inhibition are unclear (Hajdu-Cronin et al., 1999; Miller et al., 1999). Both $d g k-1$ (directly) and eat-16 (indirectly) modulate DAG levels and $d g k$-1- and eat-16-null mutants were both identified in screens for suppressors of activated $\mathrm{G} \alpha_{\mathrm{o}}$ (Hajdu-Cronin et al., 1999; Miller et al., 1999; Nurrish et al., 1999).

\section{Neuropeptides encoded by $n l p-3$ activate NPR-17 and are} essential for 5-HT stimulation

Neuropeptides are often colocalized with classical neurotransmitters, with peptides released often to increase stimulation. $n l p-3 p:: g f p$ is expressed in the ADF, NSMR and HSN serotonergic neurons, as well as many sensory neurons, suggesting that peptides encoded by $n l p-3$ are released as cotransmitters with 5-HT from the serotonergic neurons and glutamate from the sensory neurons (Nathoo et al., 2001). However, the release of $n l p-3$ peptides from the ASHs alone appears to be sufficient to stimulate ASH-mediated aversive responses. $n l p-3$ encodes three predicted peptides that were preliminarily grouped with crustacean orkokinins. However, orkokinins require a C-terminal GFxGF motif for activity that is absent in NLP-3 peptides (Nathoo et al., 2001). Attempts to heterologously express NPR-17 have been unsuccessful, but extensive genetic analyses is entirely consistent with NPR-17 being a receptor for NLP-3 peptides. For example, both nlp-3- and npr-17-null animals (1) fail to increase aversive responses on 5-HT, (2) are more sensitive to OA inhibition at elevated octanol concentrations, and (3) exhibit identical reversal phenotypes off food. In addition, wild-type animals overexpressing either $n l p-3$ or $n p r-17$ exhibit more rapid responses off food than wild-type animals that are absent when $n l p-3$ or $n p r-17$ are overexpressed in $n p r-17$ - or $n l p$-3-null animals, respectively.

ASH signaling can be operationally divided into three overlapping circuits that (1) stimulate backward locomotion via AVA/AVD command interneurons, (2) inhibit forward locomotion via AVB command interneurons and a second pathway innervating the RIMs via the AIA/AIB interneurons and (3) sensitize head muscle through the ring interneurons (RIAs) (Harris et al., 2009). A different 5-HT receptor is essential in each branch for food or 5-HT stimulation; SER-5 in the ASHs, MOD-1 in the AIBs and SER-1 in the RIAs (Harris et al., 2009). Clearly, the release of $n l p-3$-encoded peptides are also essential for 5-HT stimulation and the localization of their putative receptor, NPR-17, will be critical for understanding the role of peptidergic signaling in monoaminergic modulation. For example, 5-HT and/or NPR-17 receptors could modulate postsynaptic glutamate receptors, neuronal excitability, gap junctions and/or presynaptic neurotransmitter release.

\section{$\mathrm{G} \alpha_{\mathrm{s}}$ signaling stimulates the release of peptides encoded by nlp-3 from the ASHs}

Neurotransmitters are secreted from synaptic vesicles (SVs) that contain classical neurotransmitters, such as glutamate, and dense core vesicles (DCVs) that contain neuropeptides that activate GPCRs to modulate the sensitivity of both presynaptic and postsynaptic neurons. Although factors regulating exocytosis from SVs and DCVs are similar, the DAG-binding proteins, UNC-13 (MUNC-13-1) and UNC-31 (CAPS) play selective roles in exocytosis from SVs and DCVs, respectively, with UNC-31 involved in the docking of fusion-competent DCVs (Gracheva et al., 2007; Hammarlund et al., 2007; Speese et al., 2007; Zhou et al., 2007). G $\alpha_{\mathrm{s}}$ signaling and protein kinase A (PKA) activation enhance exocytosis from DCVs. For example, in C. elegans, PKA activation rescues the uncoordinated phenotype of $u n c-31 \mathrm{mu}-$ tants, bypasses the requirement for UNC-31 in the docking of DCVs, and directly stimulates DCV exocytosis (Charlie et al., 2006; Zhou et al., 2007).

Our data suggest that activating $\mathrm{G} \alpha$ s signaling in the ASHs stimulates peptide release that, in turn, increases ASH-mediated aversive responses. For example, (1) the more rapid responses off food associated with increased $\mathrm{G} \alpha_{\mathrm{s}}$ signaling are absent in $g s a-1 g$; 
$n l p-3$ or $p d e-4 ; n l p-3$ animals, (2) the ASH RNAi knockdown of $p d e-4$ fails to increase responses off food in $n l p-3$ animals, and (3) the ASH RNAi knockdown of $n l p-3$ in pde-4 animals abolishes increased responses observed in pde-4 animals off food. These data suggest that $\mathrm{G} \alpha_{\mathrm{s}}$-dependent sensitization of the ASHs requires the release of peptides encoded by $n l p-3$. In contrast, the increased aversive responses associated with increased $\mathrm{G} \alpha_{\mathrm{q}}$ signaling off food did not require $n l p-3$, suggesting that DCV exocytosis in the ASHs may not be essential for G $\alpha_{\mathrm{q}}$-mediated stimulation. For example, the ASH RNAi knockdown of $n l p-3$ had no effect on the increased responses off food in egl-30gf animals and the ASH RNAi knockdown of $d g k-1$ increased responses off food in both wild-type and nlp-3-null animals. Presumably, $\mathrm{G} \alpha_{\mathrm{q}}$ signaling increases the UNC-13-dependent release of glutamate from SVs.

Together, these studies demonstrate that 5-HT and OA antagonistically modulate the ASH sensory neurons and that the $\mathrm{G} \alpha_{\mathrm{s}}$ dependent release of NLP-3-encoded peptides from the ASH is essential for 5-HT stimulation.

\section{References}

Alkema MJ, Hunter-Ensor M, Ringstad N, Horvitz HR (2005) Tyramine functions independently of octopamine in the Caenorhabditis nervous system. Neuron 46:247-260.

Aspöck G, Ruvkun G, Bürglin TR (2003) The Caenorhabditis elegans ems class homeobox gene ceh-2 is required for M3 pharynx motorneuron function. Development 130:3369-3378.

Bargmann CI, Kaplan JM (1998) Signal transduction in the Caenorhabditis elegans nervous system. Annu Rev Neurosci 21:279-308.

Bargmann CI, Hartwieg E, Horvits HR (1993) Odorant-selective genes and neurons mediate olfaction in C. elegans. Cell 74:515-527.

Bergamasco C, Bazzicalupo P (2006) Chemical sensitivity in Caenorhabditis elegans. Cell Mol Life Sci 63:1510-1522.

Bloomfield SA, Völgyi B (2009) The diverse functional roles and regulation of neuronal gap junctions in the retina. Nat Rev Neurosci 10:495-506.

Brenner S (1974) The genetics of Caenorhabditis elegans. Genetics 77:71-94.

Chao MY, Larkins-Ford J, Tucey TM, Hart AC (2005) LIN-12 Notch functions in the adult nervous system of C. elegans. BMC Neurosci 6:45.

Chao MY, Komatsu H, Fukuto HS, Dionne HM, Hart AC (2004) Feeding status and serotonin rapidly and reversibly modulate a Caenorhabditis elegans chemosensory circuit. Proc Natl Acad Sci U S A 101:15512-15517.

Charlie NK, Schade MA, Thomure AM, Miller KG (2006) Presynaptic UNC-31 (CAPS) is required to activate the G alpha (s) pathway of the Caenorhabditis elegans synaptic signaling network. Genetics 172:943-961.

Chase DL, Koelle MR (2007) WormBook. Review 20:1-15.

Cohen M, Reale V, Olofsson B, Knights A, Evans P, de Bono M (2009) Coordinated regulation of foraging and metabolism in C. elegans by RFamide neuropeptide signaling. Cell Metab 9:375-385.

Colbert HA, Smith TL, Bargmann CI (1997) OSM-9, a novel protein with structural similarity to channels, is required for olfaction, mechanosensation, and olfactory adaptation in Caenorhabditis elegans. J Neurosci 17:8259-8269.

Dernovici S, Starc T, Dent JA, Ribeiro P (2006) The serotonin receptor SER-1 (5HT2ce) contributes to the regulation of locomotion in Caenorhabditis elegans. Dev Neurobiol 67:189-204.

Esposito G, Di Schiavi E, Bergamasco C, Bazzicalupo P (2007) Efficient and cell specific knock-down of gene function in targeted C. elegans neurons. Gene 395:170-176.

Gracheva EO, Burdina AO, Touroutine D, Berthelot-Grosjean M, Parekh H, Richmond JE (2007) Tomosyn negatively regulates CAPS-dependent peptide release at Caenorhabditis elegans synapses. 27:10176-10184.

Gray JM, Hill JJ, Bargmann CI (2005) A circuit for navigation in Caenorhabditis elegans. Proc Natl Acad Sci U S A 102:3184-3191.

Hajdu-Cronin YM, Chen WJ, Patikoglou G, Koelle MR, Sternberg PW (1999) Antagonism between $\mathrm{G}_{\mathrm{o}} \alpha$ and $\mathrm{G}_{\mathrm{q}} \alpha$ in C. elegans: the RGS protein EAT-16 is necessary for $\mathrm{G} \alpha_{\mathrm{o}}$ signaling and regulates $\mathrm{G} \alpha_{\mathrm{q}}$ activity. Genes Dev 13:1780-1793.

Hammarlund M, Palfreyman MT, Watanabe S, Olsen S, Jorgensen EM (2007) Open syntaxin docks synaptic vesicles. PloS Biol 5:e198.
Harris GP, Hapiak VM, Wragg RT, Miller SB, Hughes LJ, Hobson RJ, Steven R, Bamber B, Komuniecki RW (2009) Three distinct amine receptors operating at different levels within the locomotory circuit are each essential for the serotonergic modulation of chemosensation in Caenorhabditis elegans. J Neurosci 29:1446-1456.

Hawasli AH, Saifee O, Liu C, Nonet ML, Crowder CM (2004) Resistance to volatile anesthetics by mutations enhancing excitatory neurotransmitter release in Caenorhabditis elegans. Genetics 168:831-843.

Herman RK, Hedgecock EM (1990) Limitations of the size of the vulval primordium of Caenorhabditis elegans by lin-15 expression in surrounding hyopodermis. Nature 348:169-171.

Hilliard MA, Bergamasco C, Arbucci S, Plasterk RH, Bazzicalupo P (2004) Worms taste bitter: ASH neurons, QUI-1, GPA-3 and ODR-3 mediate quinine avoidance in Caenorhabditis elegans. EMBO J 23:1101-1111.

Hilliard MA, Apicella AJ, Kerr R, Suzuki H, Bazzicalupo P, Schafer WR (2005) In vivo imaging of C. elegans ASH neurons: cellular response and adaptation to chemical repellents. EMBO J 24:63-72.

Hobert O (2002) PCR fusion-based approach to create reporter gene constructs for expression analysis in transgenic C. elegans. Biotechniques 32:728-730.

Horvitz HR, Chalfie M, Trent C, Sulston JE, Evans PD (1982) Serotonin and octopamine in the nematode C. elegans. Science 216:1012-1014.

Husson SJ, Clynen E, Baggerman G, Janssen T, Schoofs L (2006) Defective processing of neuropeptide precursors in Caenorhabditis elegans lacking proprotein convertase 2 (KPC-2/EGL-3): mutant analysis by mass spectrometry. J Neurochem 98:1999-2012.

Husson SJ, Janssen T, Baggerman G, Bogert B, Kahn-Kirby AH, Ashrafi K, Schoofs L (2007) Impaired processing of FLP and NLP peptides in carboxypeptidase E (EGL-21)-deficient Caenorhabditis elegans as analyzed by mass spectrometry. J Neurochem 102:246-260.

Jansen G, Thijssen KL, Werner P, van der Horst M, Hazendonk E, Plasterk RH (1999) The complete family of genes encoding $G$ proteins of Caenorhabditis elegans. Nat Genet 21:414-419.

Kamath RS, Ahringer J (2003) Genome-wide RNAi screening in C. elegans. Methods 30:313-321.

Kaplan JM, Horvitz HR (1993) A dual mechanosensory and chemosensory neuron in Caenorhabditis elegans. Proc Natl Acad Sci U S A 90:22272231.

Kennedy S, Wang D, Ruvkun G (2004) A conserved siRNA-degrading RNase negatively regulates RNA interference in. C. elegans. Nature 427:645-649.

Komuniecki RW, Hobson RJ, Rex EB, Hapiak VM, Komuniecki PR (2004) Biogenic amine receptors in parasitic nematodes: what can be learned from Caenorhabditis elegans? Mol Biochem Parasitol 137:1-11.

Kothmann WW, Massey SC, O'Brien J (2009) Dopamine-stimulated dephosphorylation of connexin 36 mediated AII amacrine cell uncoupling. J Neurosci 29:14903-14911.

Kramer JM, French RP, Park EC, Johnson JJ (1990) The Caenorhabditis elegans rol-6 gene, which interacts with the sqt-1 collagen gene to determine organismal morphology, encodes a collagen. Mol Cell Biol 10:2081-2089.

Lanni C, Govoni S, Lucchelli A, Boselli C (2009) Depression and antidepressants: molecular and cellular aspects. Cell Mol Life Sci 66:2985-3008.

Lee RY, Sawin ER, Chalfie M, Horvitz HR, Avery L (1999) EAT-4, a homolog of a mammalian sodium-dependent inorganic phosphate cotransporter, is necessary for glutamatergic neurotransmission in Caenorhabditis elegans. J Neurosci 19:159-167.

Li C, Kim K (2008) Neuropeptides. WormBook 25:1-36.

Lu XY, Gross RE, Bagchi S, Rubin CS (1990) Cloning, structure, and expression of the gene for a novel regulatory subunit of cAMP-dependent protein kinase in Caenorhabditis elegans. J Biol Chem 265:3293-3303.

Mello C, Fire A (1995) DNA transformation. Methods Cell Biol 48:451482.

Miller KG, Emerson MD, Rand JB (1999) Goalpha and diacylglycerol kinase negatively regulate the Gqalpha pathway in C. elegans. Neuron 24:323-333.

Montell C (2005) The TRP superfamily of cation channels. Sci STKE. 2005:re3.

Mukhopadhyay S, Lu Y, Qin H, Lanjuin A, Shaham S, Sengupta P (2007) Distinct IFT mechanisms contribute to the generation of ciliary structural diversity in C. elegans. EMBO J 26:2966-2980.

Nässel DR, Enell LE, Santos JG, Wegener C, Johard HA (2008) A large pop- 
ulation of diverse neurons in the Drosophila central nervous system expresses short neuropeptide $\mathrm{F}$, suggesting multiple distributed peptide functions. BMC Neurosci 9:90.

Nathoo AN, Moeller RA, Westlund BA, Hart AC (2001) Identification of neuropeptide-like protein gene families in Caenorhabditis elegans and other species. Proc Natl Acad Sci U S A 98:14000-14005.

Nurrish S, Ségalat L, Kaplan J (1999) Serotonin inhibition of synaptic transmission: Galpha(0) decreases the abundance of UNC-13 at release sites. Neuron 24:231-242.

Perez-Mansilla B, Nurrish S (2009) A network of G-protein signaling pathways control neuronal activity in C. elegans. Adv Genet 65:145-192.

Pierce-Shimomura JT, Morse TM, Lockery SR (1999) The fundamental role of pirouettes in C. elegans chemotaxis. J Neurosci 19:9557-9569.

Pirri JK, McPherson AD, Donnelly JL, Francis MM, Alkema MJ (2009) A tyramine-gated chloride channel coordinates distinct motor programs of a Caenorhabditis elegans escape response. Neuron 62:526-538.

Reynolds NK, Schade MA, Miller KG (2005) Convergent, RIC-8 dependent $\mathrm{G} \alpha$ signaling pathways in the $C$. elegans synaptic signaling network. Genetics 169:651-670.

Rorig B, Sutor B (1996) Serotonin regulates gap junctional coupling in the developing rat somatosensory cortex. Eur J Neuurosci 8:1685-1695.

Sagasti A, Hobert O, Troemel ER, Ruvkun G, Bargmann CI (1999) Alternative olfactory neuron fates are specified by the LIM homeobox gene lim-4. Genes Dev 13:1794-1806.

Sambongi Y, Nagae T, Liu Y, Yoshimizu T, Takeda K, Wada Y, Futai M (1999) Sensing of cadmium and copper ions by externally exposed ADL, ASE, and ASH neurons elicits avoidance response in Caenorhabditis elegans. Neuroreport 10:753-757.

Schade MA, Reynolds NK, Dollins CM, Miller KG (2005) Mutations that rescue the paralysis of Caenorhabditis elegans ric-8 (synembryn) mutants activate the $\mathrm{G} \alpha_{\mathrm{s}}$ pathway and define a third major branch of the synaptic signaling network. Genetics 169:631-649.

Smith KA, Komuniecki RW, Ghedin E, Spiro D, Gray J (2007) Genes encoding putative biogenic amine receptors in the parasitic nematode Brugia malayi. Invert Neurosci 7:227-244.

Speese S, Petrie M, Schuske K, Ailion M, Iwasaki AK, Jorgensen EM, Martin
TF (2007) UNC-31 (CAPS) is required for dense-core vesicle but not synaptic vesicle exocytosis in Caenorhabditis elegans. J Neurosci 27:61506162.

Sulston J and Hodgkin J (1988) Methods. In: The nematode Caenorhabditis elegans (Wood WB, the Community of C. elegans Researchers, eds), pp 587-606. Cold Spring Harbor, NY: Cold Spring Harbor Laboratory.

Tobin D, Madsen D, Kahn-Kirby A, Peckol E, Moulder G, Barstead R, Maricq A, Bargmann C (2002) Combinatorial expression of TRPV channel proteins defines their sensory functions and subcellular localization in $C$. elegans neurons. Neuron 35:307-318.

Troemel ER, Chou JH, Dwyer ND, Colbert HA, Bargmann CI (1995) Divergent seven transmembrane receptors are candidate chemosensory receptors in C. elegans. Cell 83:207-218.

Troemel ER, Kimmel BE, Bargmann CI (1997) Reprogramming chemotaxis responses: sensory neurons define olfactory preferences in C. elegans. Cell 91:161-169.

Tsalik EL, Hobert O (2003) Functional mapping of neurons that control locomotory behavior in Caenorhabditis elegans. J Neurobiol 56:178-197.

Walker DS, Vázquez-Manrique RP, Gower NJ, Gregory E, Schafer WR, and Baylis HA (2009) Inositol 1,4,5-trisphosphate signalling regulates the avoidance response to nose touch in Caenorhabditis elegans. PLoS Genet 5:e1000636.

White JG, Southgate E, Thomson JN, Brenner S (1986) The structure of the nervous system of the nematode Caenorhabditis elegans. Philos Trans R Soc Lond B Biol Sci 314:1-34.

Wragg RT, Hapiak V, Miller SB, Harris GP, Gray J, Komuniecki PR, Komuniecki RW (2007) Tyramine and octopamine independently inhibit serotoninstimulated aversive behaviors in Caenorhabditis elegans through two novel amine receptors. J Neurosci 27:13402-13412.

Zhao B, Khare P, Feldman L, Dent JA (2003) Reversal frequency in Caenorhabditis elegans represents an integrated response to the state of the animal and its environment. J Neurosci 23:5319-5328.

Zhou KM, Dong YM, Ge Q, Zhu D, Zhou W, Lin XG, Liang T, Wu ZX, Xu T (2007) PKA activation bypasses the requirement for UNC-31 in the docking of dense core vesicles from C. elegans neurons. Neuron 56:657669 . 